ApJ Letters accepted

\title{
Blue Horizontal Branch Stars in the Sagittarius dwarf spheroidal galaxy
}

\author{
Lorenzo Monaco \\ Dip. di Astronomia, Università di Bologna, Via Ranzani 1, 4012\%, Bologna, ITALY \\ INAF - Osservatorio Astronomico di Bologna, Via Ranzani 1, 40127, Bologna, ITALY; \\ lorenzo@uqbar.bo.astro.it \\ Michele Bellazzini \\ INAF - Osservatorio Astronomico di Bologna, Via Ranzani 1, 40127, Bologna, ITALY \\ Francesco R. Ferraro \\ Dip. di Astronomia, Università di Bologna, Via Ranzani 1, 40127, Bologna, ITALY \\ and \\ Elena Pancino \\ INAF - Osservatorio Astronomico di Bologna, Via Ranzani 1, 40127, Bologna, ITALY \\ bellazzini, ferraro, pancino@bo.astro.it
}

\begin{abstract}
We report on the recovery of a Blue Horizontal Branch (BHB) population belonging to the Sagittarius Dwarf Spheroidal Galaxy (Sgr). The sequence is clearly identified in the (V, V-I) Color Magnitude Diagram (CMD) obtained for about 500,000 stars in the region of the globular cluster M 54. The BHB morphology is similar to the analogous sequence in M 54, but it is unambiguously associated with Sgr since (i) it is detected well outside the main body of the cluster, up to more than 5 tidal radii from the cluster center and (ii) the BHB stars follow the radial distribution of the other stellar populations of Sgr. This finding finally demonstrates that the Sgr galaxy hosts a significant (of the order of $\sim 10 \%$ ) old and metal-poor stellar population ([Fe/H] $]-1.3$; age $\gtrsim 10$ Gyr), similar to that of its oldest clusters (M 54, Ter 8). We also show that the Sgr BHB sequence found here is the counterpart of the analogous feature observed by Newberg et al. (2002) in the Sgr Stream, in a field more than $80^{\circ}$ away from the center of the galaxy.
\end{abstract}


Subject headings: galaxies: individual (Sagittarius) — globular clusters: individual (M 54) — stars: horizontal-branch

\section{Introduction}

It is generally recognized that the Sagittarius dwarf Spheroidal galaxy (Ibata et al. 1994, 1997 ) is currently disrupting under the strain of the Milky Way tidal field. The stars (and clusters, see Bellazzini et al. 2003a,b) lost by the Sgr dSph remain coherently aligned along the orbital path of the galaxy, forming a huge filamentary structure, the Sgr Stream, that has now been observed over the entire sky (Ibata et al. 2001a,b, 2002; Yanny et al. 2000; Ivezic et al. 2000; Newberg et al. 2002; Martínez-Delgado et al. 2001; Kundu et al. 2002; Bellazzini et al. 2003b; Majewski et al. 2003, and references therein). Therefore, the identification and definition of all the stellar components belonging to the Sgr dSph is crucial not only to understand the Star Formation History ( $\mathrm{SFH}$ ) and chemical evolution of this keystone galaxy, but also to trace its remnants in the Galactic Halo.

The Color Magnitude Diagram (CMD) of the Sgr galaxy is dominated by the classical features of a metal-rich population, with a red and extended Red Giant Branch (RGB) and a Red Clump (RC) of He-burning stars (Mateo et al. 1995; Sarajedini \& Layden 1995; Marconi et al. 1998; Bellazzini et al. 1999a,b; Monaco et al. 2002). It is now generally recognized that this dominant population has a mean age of 4-6 Gyr (Layden \& Sarajedini (2000) hereafter LS00, Monaco et al. (2002), hereafter Pap-I) and a high mean metallicity (Smecker-Hane \& McWilliam 2002; Bonifacio \& Caffau 2003, LS00, Pap-I).

The apparent absence of old and metal poor stars (OMS) in Sgr was noted since the early studies and persists, in part, at the present day. However, OMS should be present, since (a) previous generations of stars are required to enrich the medium from which the dominant metal-rich population has formed and (b) the Sgr dSph hosts at least two classical old and metal poor globular cluster (Ter 8 and M 54, see Montegriffo et al. 1998, and LS00, respectively). Hence, we have observational evidence that OMS were formed in Sgr, even if just in globular clusters. Unfortunately, the search for RGB stars bluer than the main RGB of Sgr (hence, presumably, more metal poor) has been hampered by the strong contamination by foreground stars affecting the CMD. Therefore, the available spectroscopic surveys included only a negligible fraction of stars with $[\mathrm{Fe} / \mathrm{H}]<-1$ (Smecker-Hane \& McWilliam 2002; Bonifacio \& Caffau 2003).

A Blue Horizontal Branch (BHB) sequence is easily recognizeable in the wide-field pho-

tographic CMD of Ibata et al. (1995) and it can be considered a clear indication of the 
presence of OMS in Sgr: however this population has never been further observed in any successive Sgr CCD survey. Some additional indirect clues of the existence of a metal-poor population in Sgr exist in the literature, in particular:

1. Bellazzini et al. (1999a) surveyed an isolated field in Sgr and found a star count excess in a region of the CMD similar in magnitude and color to the HB of Ter 8 . However, due to the few (23) Sgr BHB candidates, this was considered just as a supporting evidence that old and metal poor stars exist in the field of Sgr.

2. Cseresnjes (2001) analized the light curve of $~ 1800$ RR Lyrae in the Sgr galaxy. From the location of the RRd variables in the Petersen diagram he concluded that RR Lyrae in Sgr have metallicities in the range $-2.0 \lesssim[\mathrm{Fe} / \mathrm{H}] \lesssim-1.3$ (see also LS00; Cacciari, Bellazzini \& Colucci 2002). Since RR Lyrae stars are thought to trace old populations ( $\gtrsim 10$ Gyr), this result is a convincing proof that some old and metal poor stars are present in the Sgr field population. According to Cseresnjes (2001), the central density of RR Lyrae in Sgr is $\simeq 139$ stars $/ \mathrm{deg}^{2}$. Since we found (Pap-I) $\sim 2000 \mathrm{stars} / \mathrm{deg}^{2}$ belonging to the Red Clump, the RR Lyrae should account for just $\sim 6 \%$ of the whole HB population.

In this letter we use the CMD of a large area $\left(1 \times 1 \mathrm{deg}^{2}\right)$ in the main body of the Sgr $\mathrm{dSph}$ (Pap-I) to show that a significant BHB population is indeed present in Sgr. We also show that the BHB is most likely the counterpart of the analogous feature found in the Sgr Stream by Newberg et al. (2002).

\section{The Blue HB of Sgr}

The database presented in Pap-I contains V and I photometry of $\sim 490,000$ sources down to $V \sim 23$ in a $1 \times 1 \mathrm{deg}^{2}$ field centered on the globular cluster M 54, which coincides with the position of the maximum density peak of the Sgr galaxy (see Pap-I for further details).

Figure 1 shows a zoom of the HB region of the CMD, at different distances from the center of M 54. Panel (a) refers to stars contained within the tidal radius of M $54^{1}\left(r<r_{t}\right.$, where $r_{t}=7^{\prime} .5$, Trager, King, \& Djorgovski 1995). As can be seen, although the galactic foreground stars (vertical structure at $\mathrm{V}-\mathrm{I} \simeq 0.8$ ) and the main Sgr population (Red Clump at $V \simeq 18.2$ ) are easily recognizable, the population of M 54 obviously dominates the CMD.

\footnotetext{
${ }^{1}$ In the following, $r_{t}$ must always be intended as the tidal radius of the globular cluster M 54 .
} 
In particular the cluster RGB, ranging from (V, V-I) (21, 0.9) up to $(16.4,1.3)$, and the well known $\mathrm{BHB}$, at $\mathrm{V}-\mathrm{I} \lesssim 0.4$ from $V \simeq 18$ down to $V \sim 21$, are clearly evident (see also LS00 and references therein). The BHB sequence of M 54 has been enclosed in a box that is repeated in all panels.

Panel (b) shows the CMD of stars lying at $r>r_{t}$ : as expected, while the RGB of M 54 disappears, the galactic foreground and the Sgr population dominate the CMD at this distance from M 54. However, the most surprising feature is the unexpected presence of a conspicuous population of BHB (see also panels (c) and (d)). The BHB population (and in particular its brightest bulk at $\mathrm{V}<18.6)$ remains quite numerous even in the most external part $\left(3 r_{t} \leq r \leq 6 r_{t}\right)$ of the surveyed region (see panel (d)).

Figure 1 thus demonstrates that a significant BHB population is indeed present in the main body of the Sgr galaxy, as previously suggested by Ibata et al. (1995) and Bellazzini et al. (1999a). The morphology of the BHB found in Sgr is quite similar to that of M 54, although the faintest part of the sequence $(\mathrm{V}>18.6)$ appears less populated with respect to M 54 .

In order to further test the connection between the BHB population and the Sgr galaxy, we have selected two different stellar samples (shown in the left panel of Figure 2) and we have compared their radial distributions. The first sample contains stars belonging to the $\mathrm{RC}$ and therefore traces the distribution of stars belonging exclusively to the Sgr galaxy. The second contains stars belonging to the brightest part of the BHB only $(\mathrm{V}<18.6)$ to avoid spurious effects due to incompleteness and contamination by the Blue Plume. The BHB sample should therefore trace the BHB of M 54 plus the contribution of the Sgr BHB.

Figure 3 shows the cumulative radial distribution of the two adopted test samples outside $r_{t}$ (left panel) and $2 r_{t}$ (right panel). As can ben seen from both panels of Figure 3 the two samples appear remarkably similar. A Kolmogorov-Smirnov test provides a large probability $(>66 \%)$ that the two distributions are extracted from the same parent population, supporting the idea that BHB stars outside M 54 actually belong to the Sgr galaxy.

Figure 4 shows instead the cumulative distribution of the two samples for $r>3^{\prime}$, well inside the tidal radius of M 54, but still far enough from the very core of the cluster, where crowding becomes severe. The cumulative radial profile of M 54 is represented by a King model (King 1962) with $r_{c}=0^{\prime} .11$ and $\mathrm{c}=1.84$ (Trager, King, \& Djorgovski 1995). From the inspection of the figure it is then evident that, even inside $r_{t}$, the cumulative distribution of the BHB sample is significantly different from the star distribution in M 54. In fact, to reproduce the observed BHB sample distribution, we need to use a combined population. Figure 4 shows that the global distribution of BHB stars is nicely reproduced by a composite 
population with (approximately) $\sim 30 \%$ of stars contributed by M 54 and $\sim 70 \%$ by Sgr. However, a good agreement can also be achieved by varying the relative fractions by $\sim 10 \%$.

\section{Discussion}

In the previous section we report convincing evidence that a substantial population of BHB is present in the main body of Sgr. The existence of this population was missed by all previous photometric CCD surveys, probably due to an unsufficient field of view. This recovery can shed light on different aspects of the structure and the history of this disrupting stellar system.

\subsection{BHB: tracing the Old and Metal poor stars in Sgr}

The presence of a well-populated BHB in Sgr implies the presence of a substantial population of OMS in this galaxy. In fact, BHB have been, up to now, observed only in old, metal-poor stellar populations. The only exceptions are two metal-rich and very dense globular clusters belonging to the bulge (Rich et al. 1997). However, BHB stars appear spatially segregated toward the center of these clusters, suggesting a dynamical origin for these stars (Layden et al. 1999). Due to the extremely low stellar density conditions, this kind of formation channel appears quite unlikely in the case of the Sgr galaxy. Thus, with a morphology that is very similar to that of the old, metal-poor clusters associated to the Sgr: Ter $8([\mathrm{Fe} / \mathrm{H}]=-1.99)$ and M $54([\mathrm{Fe} / \mathrm{H}]=-1.55)$, the BHB sequence of Sgr is more likely composed of genuine OMS stars.

Moreover, although it is often difficult to place precise constraints on age and metallicity from the study of the HB morphology alone ${ }^{2}$, we can anyway put some limits to the age and metallicity of the BHB population by observing that stars bluer than the RR Lyrae should be at least as old and metal-poor as the RR Lyrae themselves. In particular RR Lyrae stars are known to trace old populations ( $\gtrsim 10 \mathrm{Gyr}$ ). Hence, by combining this with the results by Cseresnjes (2001), it can be concluded that the Sgr BHB population is composed by old and metal-poor stars, with ages greater than $\sim 10$ Gyr and metallicities lower than $[\mathrm{Fe} / \mathrm{H}] \simeq-1$.3.

We can also estimate which fraction of the HB stars is composed by OMS, by comparing the stellar densities of RC stars, RR Lyrae and BHB stars.

\footnotetext{
${ }^{2}$ We recall here that the $\mathrm{HB}$ morphology depends on a combination of parameters, including age and metallicity, but also less understood processes like the mass loss during the RGB phase.
} 
We select all the RC stars with $r>r_{t}$ (lower selection box in the right panel of Figure 2 ). Then we estimate the level of foreground contamination by counting stars within a box adjacent to the RC sample box (upper selection box in the right panel of Figure 2) having the same size, and we obtain $\mathrm{N}_{f} \sim 1000$ stars $/ 0.95 \mathrm{deg}^{2}$ (we consider all stars with $r>r_{t}$, i.e. a total area of $0.95 \mathrm{deg}^{2}$ ). We use this estimate to clean the RC sample, obtaining a final $\mathrm{RC}$ density of $\mathrm{N}_{R C} \sim 2000$ stars/0.95 $\mathrm{deg}^{2}$. In the same region, we counted stars belonging to the bright BHB sample defined in Figure 2, obtaining $\mathrm{N}_{B H B}=147$ stars $/ 0.95 \mathrm{deg}^{2}$. Finally, from the work of Cseresnjes (2001), the expected number of RR Lyrae is $\mathrm{N}_{R R} \simeq 130 \mathrm{stars} / 0.95 \mathrm{deg}^{2}$.

We conclude that the fraction of old and metal-poor stars in the Sgr galaxy is $\sim 12 \%$. Therefore, while the most important episodes of star formation in the Sgr galaxy occurred between 4 and 6 Gyr ago (Pap-I, corresponding ${ }^{3}$ to $\left.z<1\right)$ from a quite metal-rich $([\mathrm{M} / \mathrm{H}] \gtrsim$ 0.6) interstellar medium, a non negligible fraction of metal poor stars were already in place more than 10 Gyr ago $(z \gtrsim 2)$.

Bellazzini et al. (1999a) estimated a larger, but still compatible, fraction of OMS: $\leq 30 \%$. However it has to be noted that our estimate should be considered a lower limit since a larger fraction can be obtained if fainter BHB are taken into account.

\subsection{BHB stars in the Sgr Stream}

Recently Newberg et al. (2002), using the Early Data Release of the Sloan Digital Sky Survey, SDSS 4 , report on the discovery of an unexplained stellar population in a region of the sky named S341+57-22.5, in the direction $(l, b) \simeq(341,+57)$. The authors suggest that the unknown population is part of the Sgr Stream since (1) S341+57-22.5 lies on the predicted Sgr's orbital path (according to Ibata \& Lewis 1998; Ibata et al. 2001b) and (2) the CMD is quite similar to that of the Sgr galaxy published by Marconi et al. (1998), with the notable exception of a BHB sequence which was lacking in any previous CMD of Sgr (with the only exception of the photographic CMD by Ibata et al. 1995).

We used the same equations that Newberg et al. (2002) applied to the (V,V-I) photometry of Marconi et al. (1998) to transform our photometry into the SDSS photometric system, adopting the same distance and reddening as well. The BHB presented in this Letter is then located in the CMD at $-0.3<g^{*}-r^{*}<0.0$, a position which is fully compatible with the BHB sequence discovered by Newberg et al. (2002). Thus, the BHB population found by

\footnotetext{
${ }^{3} \mathrm{~A}$ cosmology with $h=0.65, \Omega_{\text {matter }}=0.3$ and $\Omega_{\Lambda}=0.7$ has been assumed.

${ }^{4}$ See http://www.sdss.org.
} 
Newberg et al. (2002) in the Sgr stream corresponds to the BHB population found in the Sgr galaxy. This implies that the disruption of the Sgr dSph provided a sizeable contribution to the assembly of the Galactic Halo not only in the form of metal-rich stars (traced by the M giants studied by Ibata et al. 2002; Bellazzini et al. 2003b; Majewski et al. 2003) but also providing a substantial amount of old and metal-poor stars that constitute the typical halo population.

We acknowledge the financial support from the italian Ministero dell'Università e della Ricerca (MIUR) and from the Agenzia Spaziale Italiana (ASI).

\section{REFERENCES}

Bellazzini, M., Ferraro, F.R., Buonanno, R., 1999a, MNRAS, 304, 633

Bellazzini, M., Ferraro, F.R., Buonanno, R., 1999b, MNRAS, 307, 619

Bellazzini, M., Ferraro, F.R. \& Ibata, R., 2003a, AJ, 125, 188

Bellazzini, M., Ibata, R., Ferraro, F.R., \& Testa, V., 2003b, A\&A, in press (astro$\mathrm{ph} / 0305107)$

Bonifacio, P., \& Caffau, E., 2003, A\&A, 339, 1183

Cacciari, C., Bellazzini, M., \& Colucci, S., 2002, in Extragalactic Star Clusters, IAU Symposium 207, D. Geisler, E.K. Grebel, and D. Minniti eds., San Francisco: Astronomical Society of the Pacific, p. 168

Cseresnjes, P., 2001, A\&A, 375, 909

King, I.R., 1962, AJ, 67, 471

Kundu, A., et al., 2002, ApJ, L125

Ibata, R.A., Irwin, M.J., \& Gilmore, G., 1994, Nature, 370, 194

Ibata, R. A., Gilmore, G., \& Irwin, M. J. 1995, MNRAS, 277, 781

Ibata, R.A., Wyse, R.F.G., Gilmore, G., Irwin, M.J., \& Suntzeff, N.B., 1997, AJ, 113, 634

Ibata, R.A., \& Lewis, G.F., 1998, ApJ, 500, 575 (IL98)

Ibata, R.A., Lewis, G.F., Irwin, M., Totten, E., \& Quinn, T., 2001a, ApJ, 551, 294 
Ibata, R.A., Irwin, M., Lewis, G.F., \&

Ibata, R.A., Lewis, G.F., Irwin, M. \& Cambrésy, L., 2002, MNRAS, 332, 921

Ivezic, Z., et al., 2000, AJ, 120, 963

Layden, A.C., Ritter, L.A., Welch, D.A., \& Webb, T.M.A., 1999, AJ, 117, 1313

Layden, A.C., \& Sarajedini, A., 2000, AJ, 119, 1760 (LS00)

Majewski, S.R., Skrutsie, M.F., Weinberg, M.D., Ostheimer, J.C., 2003, ApJ, submitted (astro-ph/0304198)

Marconi, G., Buonanno, R., Castellani, M., Iannicola, G., Molaro, P., Pasquini, L. \& Pulone, L., 1998, A\&A, 330, 453

Martínez-Delgado, D., Aparicio, A., Gómez-Flechoso, M.A., \& Carrera, R., 2001, ApJ, 549, L199

Mateo, M., Udalski, A., Szymansky, M., Kaluzny, J., Kubiak, M., \& Krzeminski, W., 1995, AJ, 110, 1141

Monaco L., Ferraro, F.R., Bellazzini, M., Pancino, E., 2002, ApJ, 578, L47 (Pap-I)

Montegriffo, P., Bellazzini, M., Ferraro, F.R., Martins, D., Sarajedini, A., \& Fusi Pecci, F., 1998, MNRAS, 294, 315

Newberg, H.J., et al., 2002, ApJ, 569, 245 (N02)

Rich, R.M., et al., 1997, ApJ, 484, L25

Sarajedini, A., Layden, A.C., 1995, AJ, 109, 1086

Smecker-Hane, T.A., \& McWilliam, A., 2002, ApJ, submitted (astro-ph/0205411)

Trager, S. C., King, I. R., \& Djorgovski, S. 1995, AJ, 109, 218

Yanny, B., et al., 2000, ApJ, 540, 825 


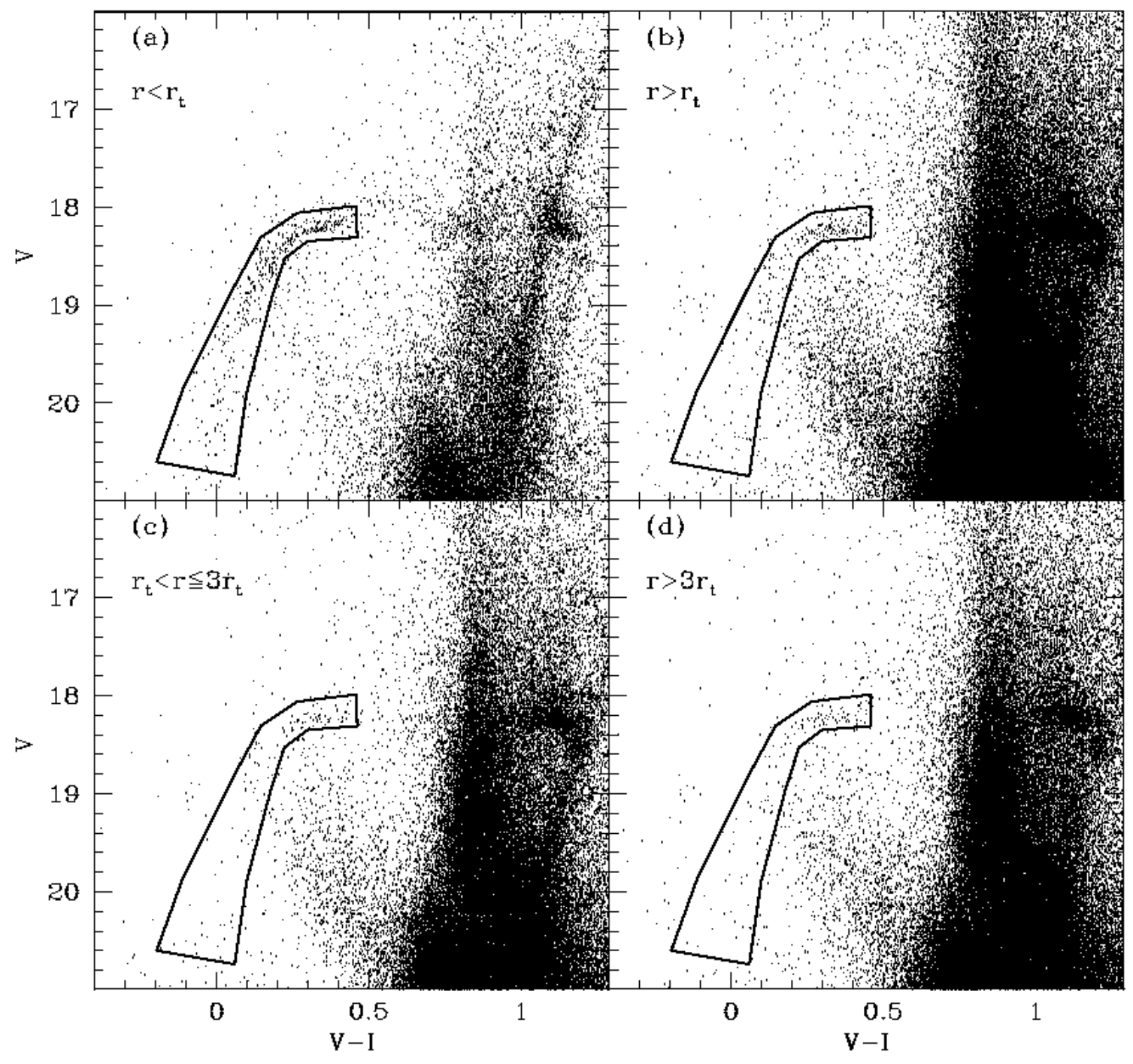

Fig. 1.- CMDs at different radial distances from the center of the globular cluster M 54 . The thick line encloses stars belonging to the BHB in all panels. 


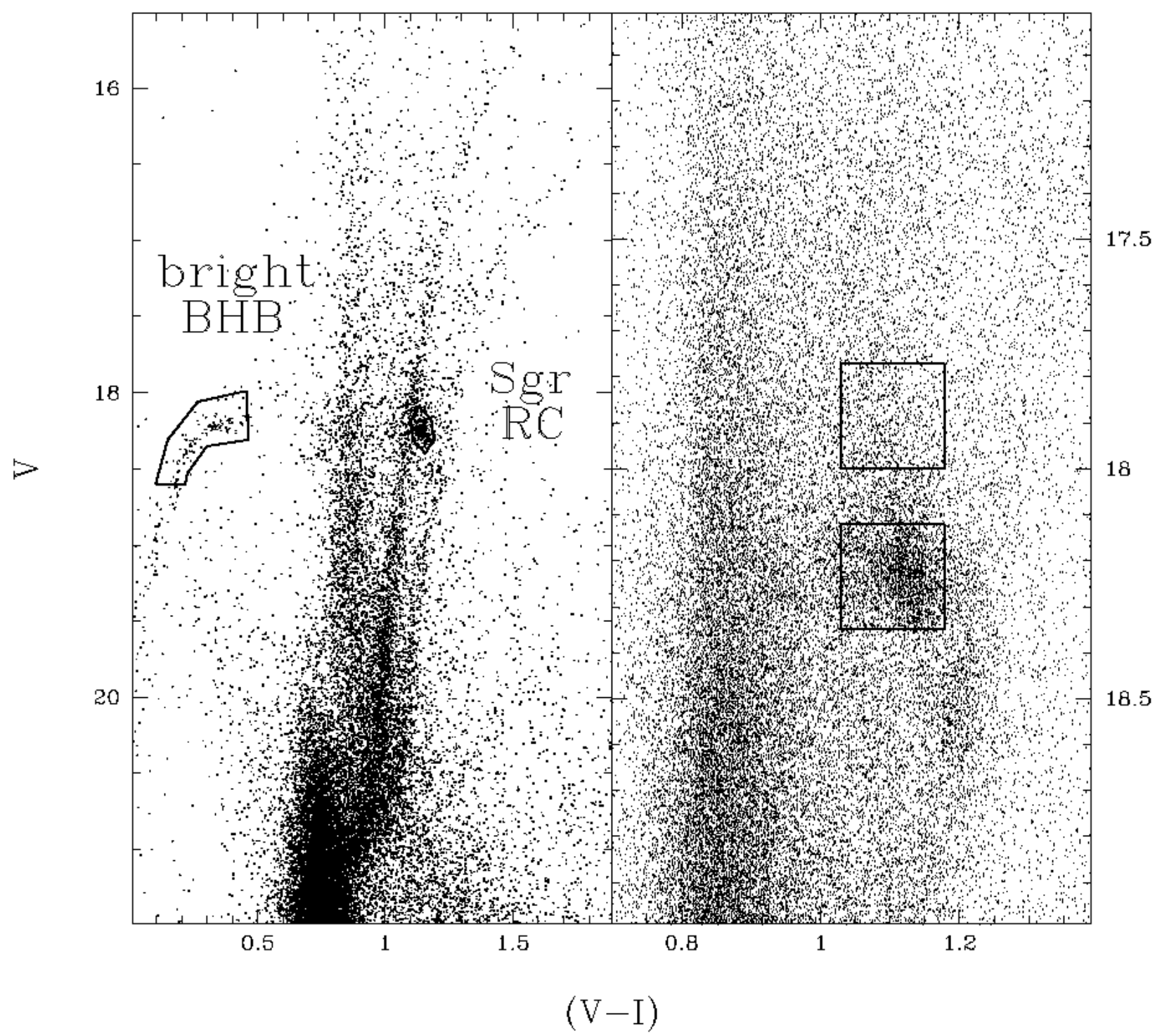

Fig. 2.- Left panel: adopted selection boxes for BHB and RC stars as tracer populations on the Sgr CMD. Right panel: CMD of Sgr outside the tidal radius of M 54. The lower box encloses stars in the RC phase, while the upper selection box is used for field decontamination. 




Fig. 3.- The cumulative radial distribution of the BHB sample (solid line) is compared with that of the RC control sample (dotted line), outside $r_{t}$ (left panel) and $2 r_{t}$ (right panel) from M 54 . 


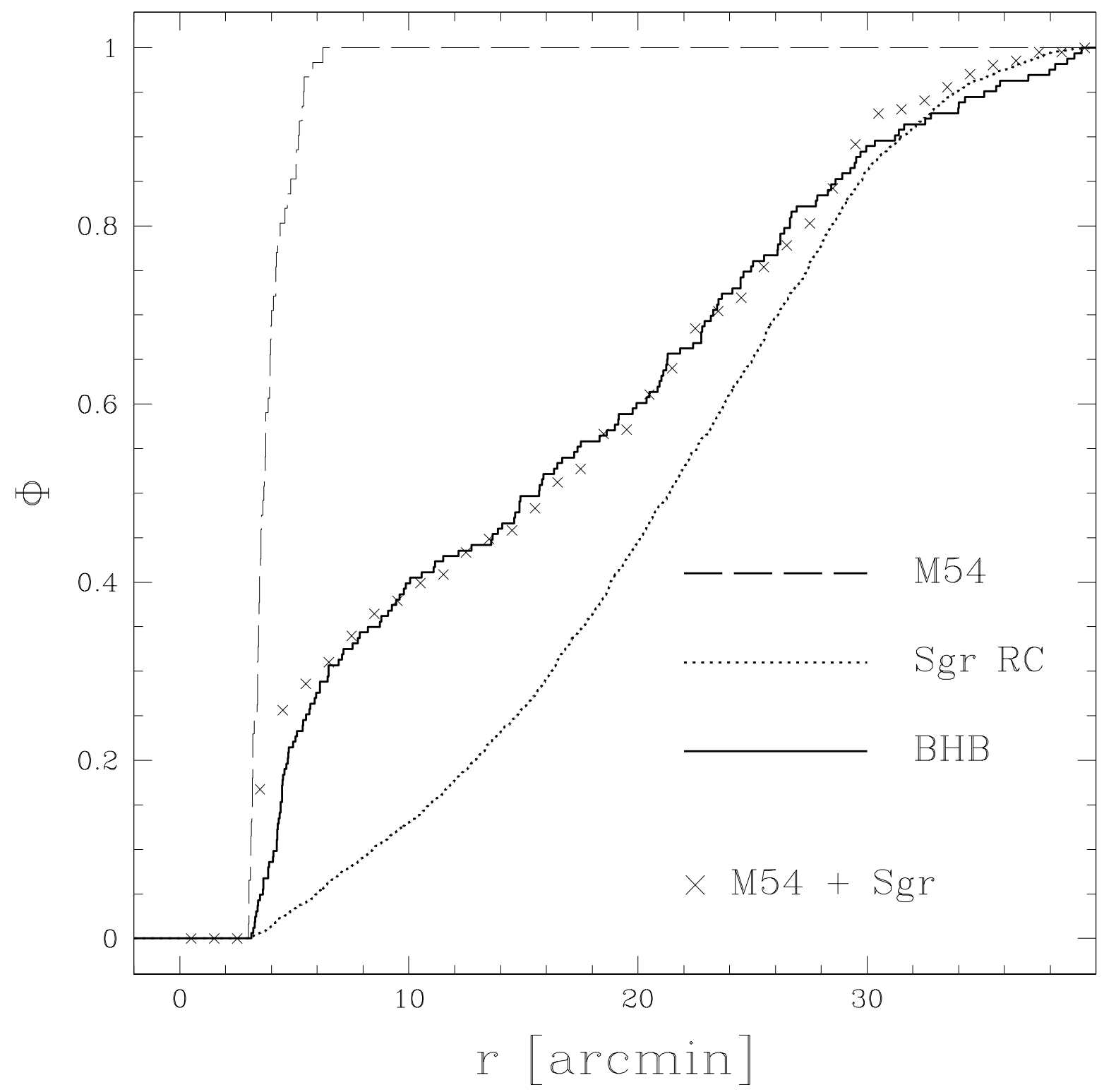

Fig. 4.- The cumulative radial distribution of the BHB sample (solid line), starting at $3^{\prime}$ from the center of M 54, is compared with the distribution of the RC sample (dotted line) and of M 54 (dashed line), represented by its King profile (King 1962) with $r_{c}=0^{\prime} .11$ and $\mathrm{c}=1.84$ (Trager, King, \& Djorgovski 1995). Crosses represent a suitable combination of the profile of M $54(\sim 30 \%)$ and that of the Sgr dSph ( 70\%). 